\title{
Period analy tic algorithms for the estimation of selected spectral properties of short segments of EEG data*
}

\author{
JACKSON BEATTY and CARL FIGUEROA \\ The University of California, Los Angeles, California 90024
}

\begin{abstract}
The accuracy and computational speed of three algorithms (spectral analysis, period analysis, and period amplitude analysis) for the extraction of spectral estimates from time-series data are empirically evaluated in the context of a neuropsychological experiment that requires estimates of power in the standard electroencephalographic frequency bands.
\end{abstract}

Computer analysis techniques have expanded the range of possible tools available to the investigator in the psychological and physiological laboratory. Of particular interest to the neuropsychologist are the capacity for real-time signal analysis in either the time or the frequency domain and the possibility of response-contingent control of experimental events. We have employed a general-purpose digital computer to analyze short segments of EEG data and to generate response-contingent reinforcement on the basis of various parameters of EEG activity (Beatty, 1971, 1972; Beatty \& Kornfeld, 1972). In these investigations, a zero-crossing algorithm was employed to estimate percent spectral power in the alpha (8-12) and beta $(12-30 \mathrm{~Hz})$ frequency bands. While the theoretical correspondence of period analytic with spectral analysis techniques based upon the Fourier transform is known (Saltzberg, 1973), little is known about the stability of these measures when the length of the time series is extremely limited. Since our experimental paradigm required the estimation of spectral parameters of $1-\mathrm{sec}$ segments of EEG data, an evaluation of the correspondence of these two algorithms for short data segments was of interest to us and is the subject of this report.

Electroencephalographic data were obtained from five undergraduate volunteer Ss, whose only task was to sit quietly in a lighted room. EEG was recorded from a monopolar occipital configuration through a Grass P-15 preamplifier and a series of integrated circuit amplifiers and active filters (system bandpass: $3 \mathrm{~dB}$ attenuation at 1 and $40 \mathrm{~Hz}$ ). Fifty $1.024 \mathrm{sec}$ records of EEG were obtained from each S, yielding 250 records for the group. Each such record was composed of 1,024

*This research was supported by the Advanced Research Projects Agency of the Department of Defense and was monitored by the Office of Naval Research under Contract No001-70-C-0350. Additional on-line computer processing for data analysis was provided by the Center for Computer-based Behavioral Studies under Contract F30602-70-C0016 with Advanced Research Projects Agency of the Department of Defense. samples. After acquisition, each record was displayed for the $\mathrm{E}$ on a Tektronix 601 storage unit. If there was any evidence of movement artifact in the record, the record was discarded and replaced. The 250 records were stored separately on disk for subsequent analysis.

Using either the conventional Fourier transform or the rapid processing algorithm (fast Fourier transform) developed by Cooley and Tukey (1965), time series data may be transformed into the frequency domain with no loss of information. From the resulting real and imaginary Fourier coefficients, estimates of power and phase angle of the signal at multiples of the fundamental frequency may be obtained. Although these procedures are rather complicated, they are now well understood, and standardized computational procedures have been published (Jenkins \& Watts, 1968; Welch, 1967).

In the present application, the initial time series records were decimated by a factor of 4 , yielding corrected time series of 256 points sampled at a rate of $250 \mathrm{~Hz}$. Spectral analysis of these data produced estimates of power from 0 to $125 \mathrm{~Hz}$ in 129 frequency bands $.97 \mathrm{~Hz}$ wide. This spectrum was collapsed into six bins corresponding to the standardized electroencephalographic frequency bands, with adjustment at the boundaries when necessary. Phase information was discarded.

Full wave period analysis was accomplished by measuring the interval in milliseconds between alternate primary zero crossing in the digitized raw EEG. Each wavelength measure obtained was classified into one of the six standard electroencephalographic frequency bins and its occurrence marked in the appropriate counter. Thus, each record resulted in a profile of counts corresponding to the wavelengths measured for that record.

A second period-based algorithm utilizes amplitude information. In period-amplitude analysis, measurement of the interval between positive-going primary zero crossings was accomplished as above. Amplitude information was introduced by summing the root mean 


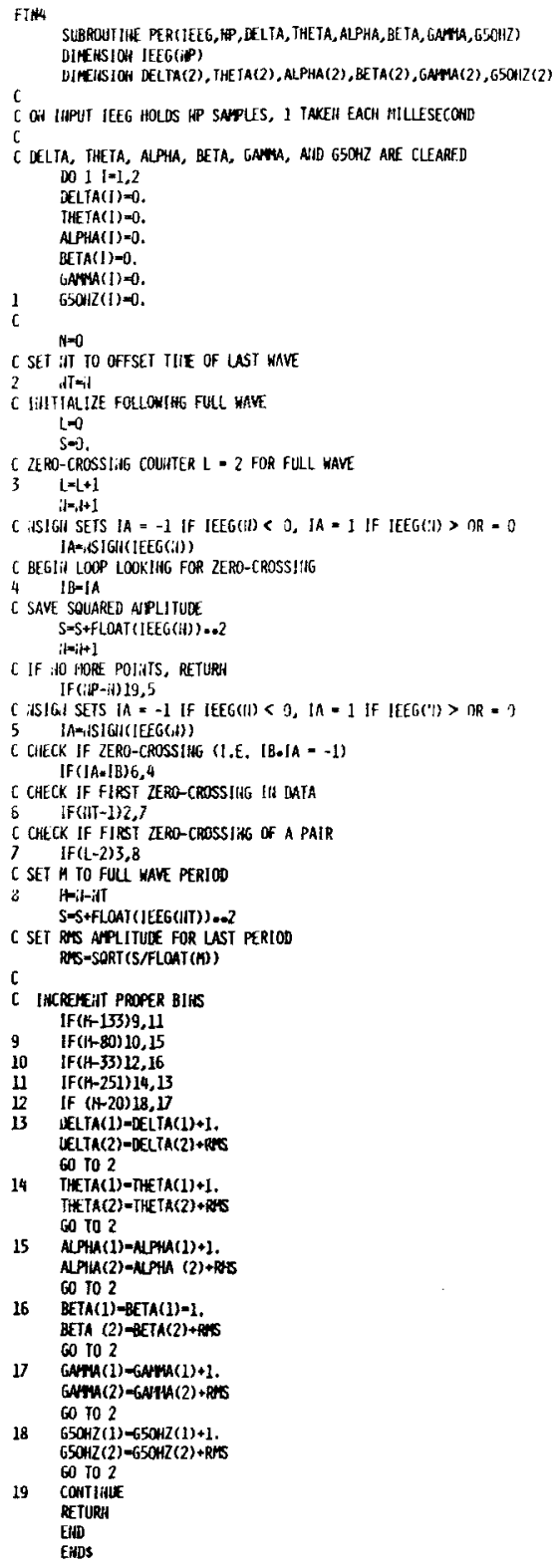

Fig. 1. A FORTRAN IV subroutine for period and period-amplitude analyses of EEG data.

squared amplitude of each wave into the appropriate frequency bin. Thus, the algorithm acted to integrate the power contained in each full wave of the EEG. Each record was represented as a profile of the power present in the various frequency bands.

A subroutine incorporating both the period and period-amplitude analyses is presented for instructional purposes in Fig. 1. Although configured for processing the stored data base, this code was developed for real-time analysis. There, EEG data was digitized once each millisecond as a 10-bit word and hard clipped by the NSIGN function to prevent integer overflow upon multiplication of adjacent values in the test for zero crossings, indicated by a negative product. The remaining code measures the full wave period between alternate zero crossings, incrementing the appropriate counter in period analysis and summing the rms amplitude of the EEG of that period in the period-amplitude analysis.

Although, for signals of sufficiently wide bandwidth, computation time would constrain the use of period analytic methods, the spectral properties of EEG data present no serious difficulties. Nearly all power in the EEG is below $50 \mathrm{~Hz}$, suggesting that a millisecond sampling period is more than sufficient (since the Nyquist frequency is exceeded by a factor of 10). Even at a $1,000-\mathrm{Hz}$ data rate, sufficient time exists in the dedicated computer to acquire data values from one or several data channels, to check each channel for a zero crossing, and to update whatever tables are necessary on the basis of that information. At the completion of the analysis epoch, all that remains is to summarize the tabulated data in some fashion, typically involving only a few divisions and multiplications, and transmitting the estimates obtained to the appropriate portion of the program. The period amplitude algorithm requires little more computation time; it is necessary to square the voltage values upon acquisition and obtain the root of their average on the detection of an appropriate zero crossing. Thus, either algorithm easily meets the real-time constraints on analysis that are present in many experimental electrophysiological paradigms.

Each of the 250 data records was analyzed using spectral, period, and period-amplitude analyses. The results of each analysis were converted to estimates of the standard electroencephalographic spectral categories: delta $(.25-4 \mathrm{~Hz})$, theta $(4-7 \mathrm{~Hz})$, alpha $(8-12 \mathrm{~Hz})$, beta $(13-30 \mathrm{~Hz})$, and gamma $(31-50 \mathrm{~Hz})$ activity for each record. In addition, the presence of activity above $50 \mathrm{~Hz}$ was also estimated.

Each of the three methods of analy sis yields similar estimates of the averaged spectral shape of the 250 records. Figure 2 presents percent activity in each of the standard EEG bands, as estimated by period, period-amplitude, and spectral analyses. It appears that the simpler period and period-amplitude methods may be used to estimate spectral properties over a large data base.

The performance of these algorithms on limited data samples may be indexed by the rank order correlation between period and spectral parameters of the 250 individual 1.024-sec EEG records. A Spearman rank-order correlation coefficient (Siegel, 1956) was used to estimate the congruence in ordering of intervals produced by the spectral and period analytic measures. A high degree of congruence suggests that the simpler 

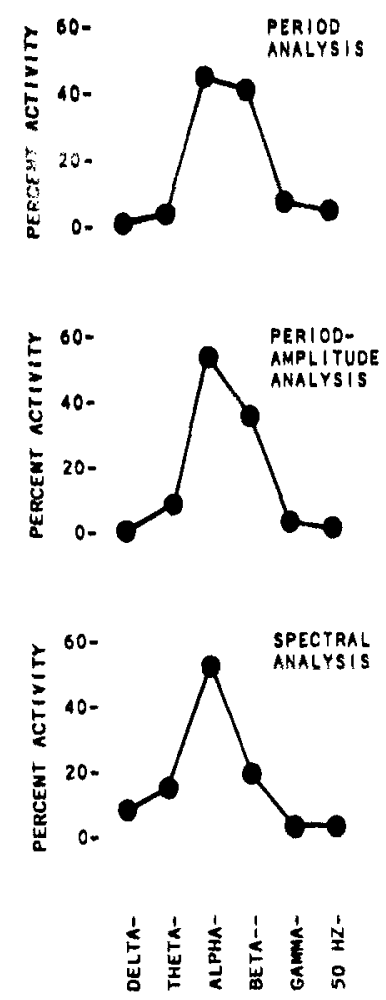

Fig. 2. Percent EEG activity in each of the standard EEG frequency bands over $250 \quad 1.024$ - $\mathrm{sec}$ data records by period, period-amplitude, and spectral analyses.

procedures may be safely employed to obtain an ordering of intervals based on the relative prominence of activity within a particular frequency band, necessary in studies involving EEG conditioning.

Period analytic methods effectively estimate the percent power in the EEG bands of major interest. Percent alpha, as measured using standard spectral methods, correlates .79 with the period and .82 with the period-amplitude estimates of the same percentages. Similarly, the correlations for percent beta frequency activity are .78 and .81 . Thus, it appears that the simpler period analytic methods may be used to estimate the percentage of spectral power in the alpha and beta frequency bands of the waking human EEG.

\section{REFERENCES}

Beatty, J. Effects of initial alpha wave abundance and operant training procedures on occipital alpha and beta wave activity. Psychonomic Science, 1971, 23 197-199.

Beatty, J. Similar effects of feedback signals and instructional information on EEG activity. Physiology \& Behavior, 1972, 9, 151-154.

Beatty, J., \& Kornfeld, C. Relative ind ependence of conditioned EEG changes and cardiac and respiratory activity. Physiology \& Behavior, 1972, 9, 733-736.

Carrie, J. R. G \& Frost, J. D., Jr. A small computer sy stem for EEG wavelength-amplitude profile analysis. Biomedical Computing, 1971, 2, 251-263.

Cooley, J, W. \& Tukey, J. W. An algorithm for the machine calculation of complex Fourier series. Mathematics of Computation, 1965, 19, 297-301.

Jenkins, G. M., \& Watts, D. G. Spectral analysis and its applications. San Francisco: Holden Day, 1968.

Saltzberg, B. Period analysis. In M. Matousek (Ed.), Evaluation of bioelectrical data from brain, nerve and muscle, II. Part $A$. Frequency and correlation analysis. In A. Reymond (Ed.), Handbook of electroencephalography and clinical neurophysiology. 5 A. Amsterdam: Elsevier, 1973.

Siegel, S. Nonparametric statistics for the behavioral sciences. New York: McGraw-Hill, 1956.

Welch, P. D. The use of fast Fourier transform for the estimation of power spectra: A method based on time averaging over short-modified periodograms. IEEE Transactions on Audio Electroacoustics, AU-15, 1967, 70-73.

\title{
A minicomputer program for stimulus control and analog data for discrete trial paradigms in biological preparations: Classical conditioning*
}

\author{
ROBERT W. TAIT and I. GORMEZANO \\ University of lowa, Iowa City, lowa 52240
}

\begin{abstract}
The application of computer technology to classical conditioning requires: control software that will repeatedly generate stimulus events, and analog-to-digital (A/D) recording capabilities for the resolution of analog data from phasic response systems (e.g., nictitating membrane extension, leg flexion). An earlier software package implemented on a 4K PDP-8/E (Millenson, Kehoe, Tait, \& Gormezano, 1973) successfully met these requirements. However, the program's capabilities were restricted to the presentation of only two unique stimuli with a maximum of 4 sec duration, and data reduction in only uniphasic response systems. The present program, employing the same hardware, relaxed these previous constraints and extended the range of data analysis through the revamping of the interactive assembler, operating system, and on-line data processing routines.
\end{abstract}

*The creation of the program and the preparation of this report were supported by NSF Grant GB-41531.
The E's operations for classical conditioning can be conceptualized as involving two types of temporal 